\title{
Erratum: Native point defects and impurities in hexagonal boron nitride [Phys. Rev. B 97, 214104 (2018)]
}

\author{
L. Weston $\odot$, D. Wickramaratne, M. Mackoit, A. Alkauskas $\odot$, and C. G. Van de Walle
}

(Received 31 August 2020; published 22 September 2020)

DOI: 10.1103/PhysRevB.102.099903

Upon reexamining our results, we discovered four inconsistencies in our earlier paper, which we have now corrected as outlined below. The corrections amount to quantitative shifts in some of the results, but none of the discussions or conclusions of the paper are impacted.

1. van der Waals interactions. In Sec. II A of the original paper, it was incorrectly stated that the Grimme-D2 scheme was used. All of the calculations in the original paper used the Grimme-D3 scheme [1].

2. Formation energies of native defects. According to Eq. (1) of the original paper, the difference in formation energies (calculated at the same Fermi energy) under N-poor and N-rich conditions is $\Delta H_{f}(\mathrm{BN})$ in the case of vacancies and selfinterstitials, and $2 \Delta H_{f}(\mathrm{BN})$ in the case of antisite defects. The difference between N-rich and N-poor conditions in Fig. 3 of the original paper reflected the experimental rather than the theoretical value of $\Delta H_{f}(\mathrm{BN})$. Because the calculated enthalpy of formation, $\Delta H_{f}(\mathrm{BN})=-2.9 \mathrm{eV}$, is close to the experimental value of $-2.6 \mathrm{eV}$, this amounts to differences by $\pm 0.3 \mathrm{eV}$. In Fig. 1 of this Erratum, we show the corrected results. Formation energies of $\mathrm{N}_{i}, V_{\mathrm{N}}$ (under N-rich conditions) and of $\mathrm{B}_{i}, V_{\mathrm{B}}$ (under N-poor conditions) remain unchanged, whereas formation energies of other defects are altered by $\pm 0.3 \mathrm{eV}$. Charge-state transition levels remain unaffected.

3. Formation energy of $\mathrm{O}_{i}$. The formation energy of $\mathrm{O}_{i}$ under N-poor conditions was incorrectly reported in Fig. 6(d) of the original paper; it should be lower by $2.3 \mathrm{eV}$. All other formation energies in Fig. 6 are unaltered. For ease of reference, in Fig. 2 of this Erratum we provide formation energies for all oxygen-related defects as a replacement for Figs. 6(c) and 6(d) of the original paper.

4. Charge-state transition level of $\mathrm{C}_{\mathrm{N}}$. The value of the $(0 /-)$ charge-state transition level of $\mathrm{C}_{\mathrm{N}}$ was incorrectly reported as $3.19 \mathrm{eV}$ in Fig. 7 of the original paper. The correct value of the $(0 /-)$ level is $2.84 \mathrm{eV}$ as cited in the main text and consistent with the formation energy diagrams in Figs. 6(a) and 6(b) of the original paper. A corrected figure depicting all charge-state transition levels is shown as Fig. 3 of the present Erratum. The results for all other defects are unchanged.

We thank Dr. M. Maciaszek for pointing out inconsistencies in the original paper. We acknowledge M. Turiansky for technical help, and A. Rowberg for discussions.
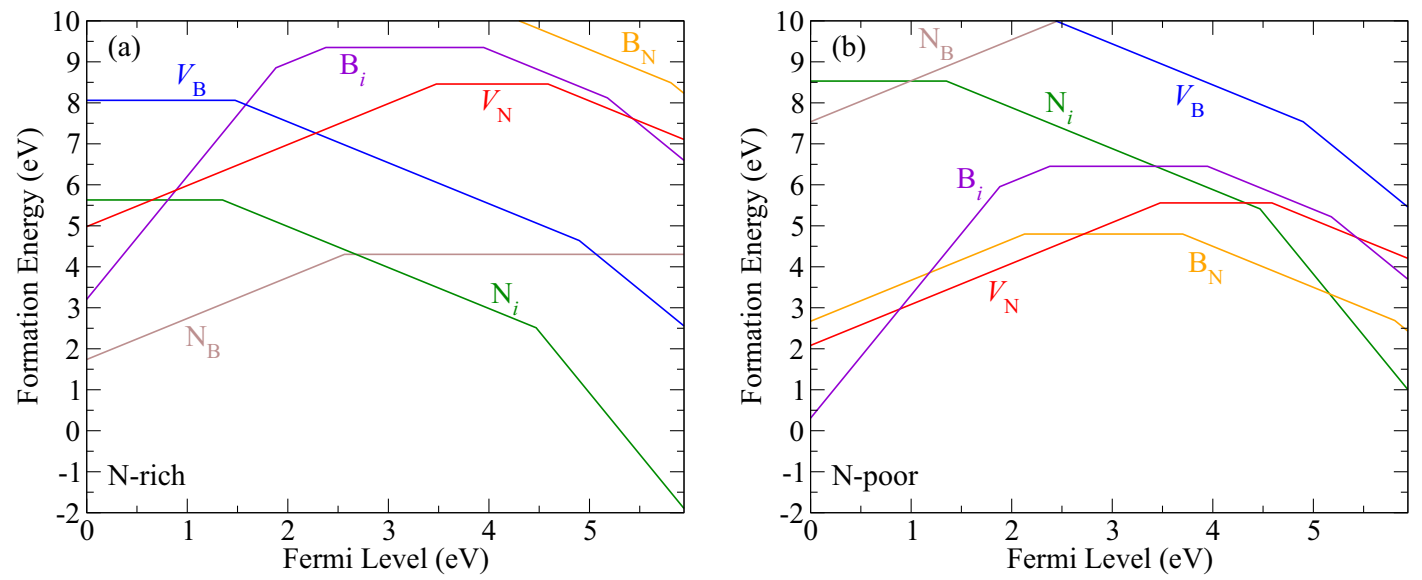

FIG. 1. Formation energies of native point defects in $h$-BN as a function of Fermi level under (a) N-rich and (b) N-poor conditions. Replaces Fig. 3 of the original paper. 

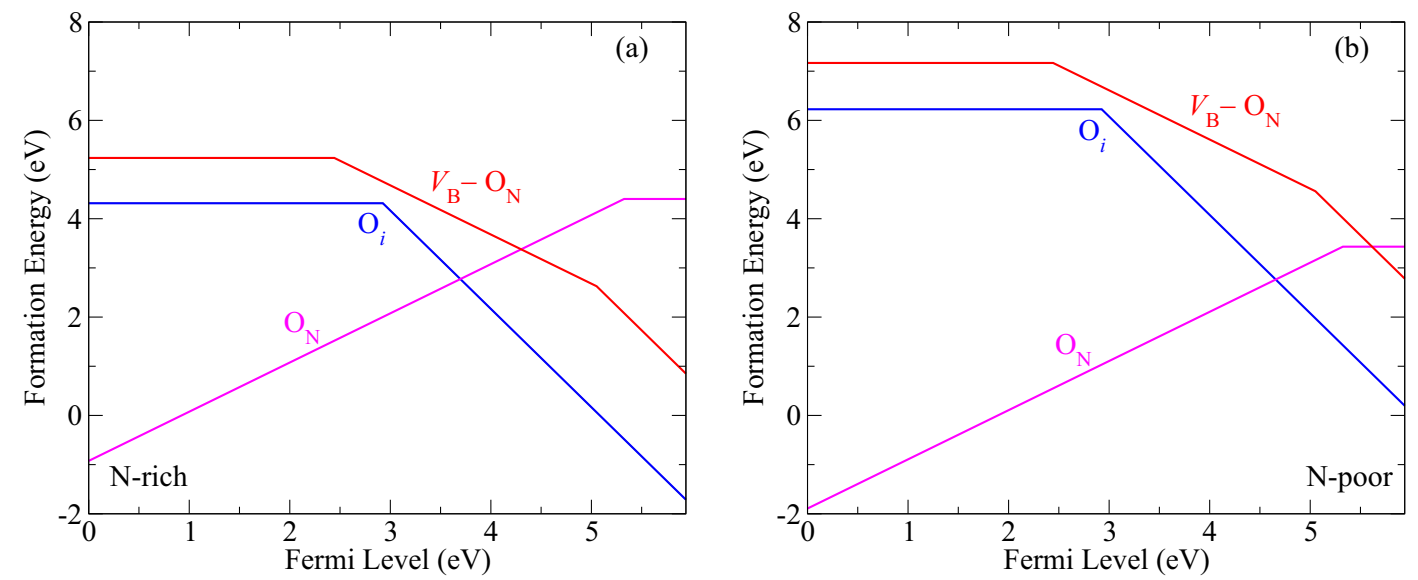

FIG. 2. Formation energies of oxygen-related defect centers in $h$-BN as a function of Fermi level under (a) N-rich and (b) N-poor conditions. Replaces Figs. 6(c) and 6(d) of the original paper.

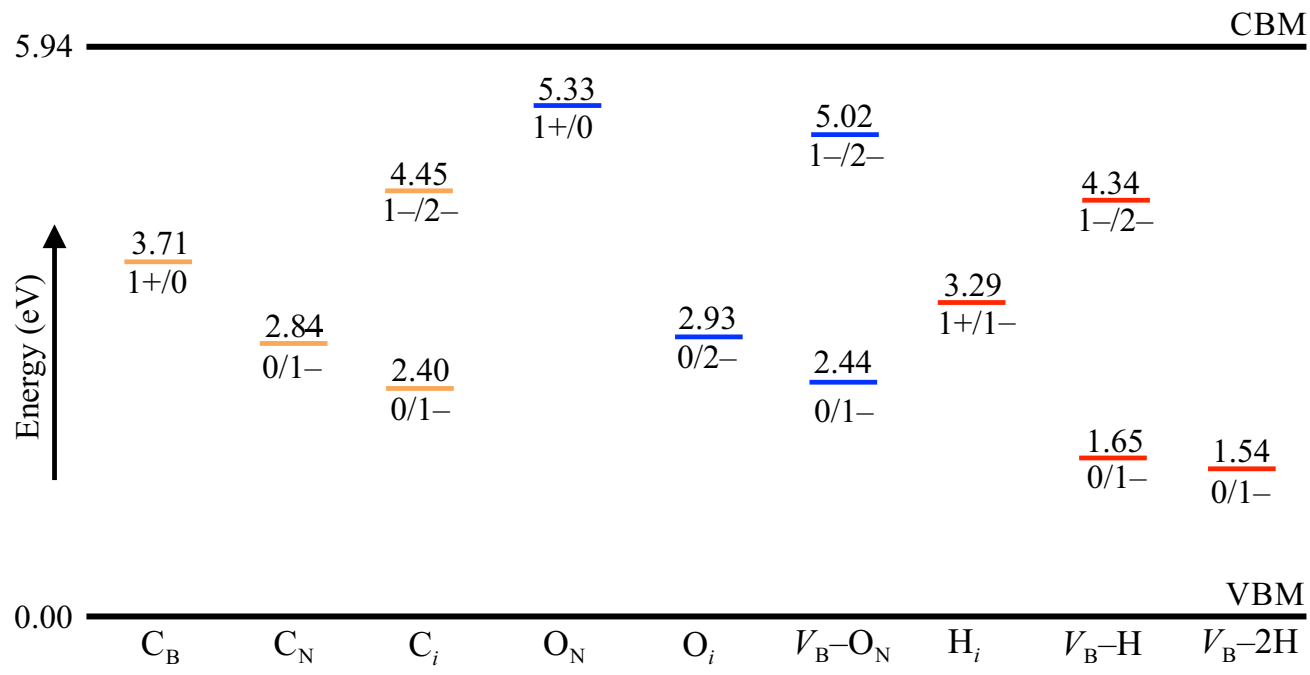

FIG. 3. Charge-state transition levels for impurity-related defect species under $h$-BN. Defect levels related to carbon are shown in orange due to oxygen in blue and hydrogen in red. Replaces Fig. 7 of the original paper.

[1] S. Grimme, J. Antony, S. Ehrlich, and H. Krieg, J. Chem. Phys. 132, 154104 (2010). 